venting her from earning a livelihood, it can be cured by removing the ovaries. This is so grave a measure, that it should not be done without most careful consideration by the patient as well as by her doctor. The risk to life of the operation is trifling. The reason against it is that it destroys the possibility of maternity, and lessens the patient's prospect of marriage. The younger she is the greater is the injury thus done her, and the less she will be able to rightly understand its extent. Hence the proposition of this remedy should be postponed as long as possible, and its effects should be fully explained to the patient through her nearest female relative, or some female friend in whom she has contidence.

\section{THE PROBLEM OF THE PREMATURE INFANT.*} By J. W. BALLANTYNE, M.D., F.R.C.P.Edin., F.R.S.EDIN.

Lecturer on Midwifery and Gynaecology, Medical College for Women, Edinburgh.

THE premature infant is born with the skin and the skeleton, and the organs of a seven-months fetus. He is called upon to play the part of a newborn infant with the personalia of a fetus. He is admirably fitted to continue living in the uterus, but is ill provided to meet the exigencies of an extrauterine existence. He is suddenly forced into surroundings of a kind which impose upon him urgent calls to which he is little able to respond. His tissues have not had time to mature, and he is not ready for so complete a change in environment. He is like some dweller in the hot plains of India who has been transported in a moment of time on some "magic carpet of Tangu" to the chill summits of the "frosty Caucasus; "with no opportunity for acclimatization such as a gradual transit affords; he is suddenly submitted to the severe strain which so marked a change in surroundings entails; it is possible that the marvellous adaptive mechanisms of the human body will overcome the difficulties of adjustment of capabilities to requirements, but there will be danger till this condition of physiological equilibrium is reached. So the premature infant is quickly transferred from the warm fluid medium of the liquor amnii with its practically constant temperature to the variable circumstances of an extrauterine life; he loses the preparatory transition-time of the third trimester of intrauterine existence, and although he may react with extraordinary success to his new environment, yet in many instances he fails to do so, and pays the heavy penalty of premature death. In too many cases there is a defective adjustment of capabilities to requirements ; this is almost inevitable, for the requirements are exacting and the capabilities inefficient; he may then escape death only to carry a debility with him which projects a baneful influence far into his postnatal life. This is the danger he runs, this is the price he has to pay for his earlier advent into the life that is after birth. Essentially the error is one of the time relations; if he had been a little longer in the uterus he had been better able to withstand the trying changes of birth ; but he is an untimely birth. The problem is one of adaptation, of utilization of manifestly imperfect powers to obtain results which are not easily to be got. Life is full of such problems; the individual is often being brought into circum. stances to which he finds it difficult and sometimes impossible to adapt himself; he is often called upon to discharge duties which he could have done better had he had a little longer for preparation. The strain and stress of life lie in these emergencies, and happily the requisite strength often emerges to overcome them; but the problem of the premature infant is peculiarly urgent, for he is so dependent upon conditions external to himself. Upon the obstetrician falls the responsibility of aiding the prematurely born child in his struggle with the exigencies of his new environment. The more thoroughly he understands the problem the more effective will his management of it prove. Let me therefore endeavour to set forth in detail the conditions which I have briefly indicated above.

VARIABILITY OF VIABILITY.

The premature infant differs from the immature fetus in the possession of viability, that is to say. he is canahle of an * Read before the Edinburgh Obstetrical zociety. independent existence outside the mother's uterus, an existence, it must be added, which is not limited to a few hours, but is potentially possible for months and years. Not only must his heart beat, his limbs move, and his lungs respire, but his digestive organs must also be capable of a certain amount of functional activity, and his tissues be able to assimilate nourishment brought to them through the alimentary canal. So much is certain; but when attempt is made to fix the uterine age at which viability may be said to be acquired difficulties are at once encountered. It is not easy, for instance, exactly to estimate the uterine age of any fetus; consequently, when one speaks of a premature infant born at the seventh month of pregnancy, one can only mean that the maternal symptoms and the physical examination of the mother and the infant pointed to that as the most probable date. Neither fetal size nor weight can be regarded as sure indications of fetal age, and the knowledge we possess of the chronology of the developmental processes is not exact enough to permit the drawing of accurate conclusions from them. Another difficulty exists in the fact that the maternal health during pregnancy has an influence upon the viability of the infant; thus, the seven-months fetus of a healthy mother may be altogether viable, whereas the seven.months fetus of a syphilitic mother may be absolutely non-viable. The age of attainment of viability will, therefore, vary with the state of health of the parents. Even if the exact uterine age of the premature infant could be determined with sufficient accuracy, there would still be the varying factor of the infant's vitality as influenced by the varying circumstances of intrauterine health; the viability of the child depends, therefore, not only upon the number of months spent in the uterus, but also upon the character of the life of these months.

It may, however, be asked whether it is not possible to fix some date which will take into account these factors of variability and give what may be regarded as a reasonable estimate of the uterine age when viability is attained. Let us, for instance, maintain that a fetus born of healthy parents, showing no obvious morbid state-either of disease or of deformity-and having an estimated age of $6 \frac{1}{2}$ calendar months, is viable. Such an assumption is perfectly reasonable, and will be accepted by every obstetrician; yet the statement cannot be regarded as final, for in ten years it may be pussible to make a similar one with respect to the fetus of 6 or even of $5 \frac{1}{2}$ months. Improvements in the management of premature infants, such as have been made possible by the introduction of the incubator and the exclusion of sepsis, have already, so to say, pushed the age of viability back; it is probable that a better understanding of the problem to be solved may lead to the devising of therapeutic, dietetic, and hygienic measures which will push it still further back. Be this as it may, it is a fact of prime importance to remember that the age of viability is not a fixed but a variable date.

The variability of viability need not, however, be interposed here to delay the discussion of the problem of the premature infant, for in this contribution the type of prematurity which is considered is that of the infant expelled from the uterus at the seventh month of intrauterine life. Every one will admit the viability of a seven-months fetus. The difficulties such an infant encounters and the dangers he runs may be regarded as the average difficulties and dangers of prematurity; they will be fewer in the eight-months and greater in the six-months fetus, for of course prematurity passes by degrees into maturity at the ninth month of intrauterine life, and merges into immaturity at the fifth.

Anatomy of the Premature Infant.

The premature infant, as has been already stated, possesses the skin and the skeleton and the internal organs of a sevenmonths fetus; but it is necessary to enter a little more into details, and to define the anatomical characters which are contained in this brief generalization. The weight of the premature infant varies from 3 to $4 \frac{1}{2} \mathrm{lb}$, and the length is about 16 in. The skin has a delicate, almost transparent, appearance, but has lost the bright red colour of earlier fetal life; there is little subcutaneous tissue, so that the face has still a somewhat senile look, and the outlines of the limbs and trunk are sharp and not rounded. The lanugo plentifully covers the extensor surfaces of the limbs as weli as the face 
and back ; there is now some hair on the scalp. The nails are thin and do not project beyond the finger tips, but they are quite horizontal as regards the underlying skin. In the eyes traces of the membrana pupillaris may still be seen, and the cranial fontanelles and sutures are large and wide. In the male infant the testicles will probably be found in the scrotum; but in a certain proportion of cases one of them may still be in the inguinal canal, and may be capable of being pressed back into the abdominal cavity. In the female infant the imperfect state of development of the labia will give to the vulva a gaping appearance.

These peculiarities of the anatomy of the premature infant are evident on external examination, but there are others which are revealed only by dissection or section. In the skeleton, nearly all the ossific nuclei which are found in the full-time infant are present in the premature one, but they are smaller in size both absolutely and relatively. The ossific nucleus in the lower epiphysis of the femur, that distinctive "blood-red spot in the midst of milk-white cartilage," is rarely to be seen, and there is none in the upper end of the tibia, in the cuboid, or in the hyoid bone. The brain shows very clearly its premature state, for its grey and white matter cannot yet be differentiated, its ventricles are relatively large, and its fissures and convolutions slightly marked. The island of Reil is still exposed to view, for the margins of the fissure of Sylvius have not approximated sufficiently to cover it in. The filum terminale of the spinal cord ends opposite the third lumbar vertebra instead of opposite the first or second, as in the full-time infant. The foramen ovale of the heart is widely open and the ductus arteriosus quite patent. The kidneys are distinctly lobulated, and the suprarenals and thymus are relatively large. The lungs, even in the premature infant that has breathed, are partly atelectatic. The caecum is seldom in its permanent position in the right iliac fossa. In other respects the anatomy of the premature infant resembles that of the mature fetus.

Anatomically, then, the premature infant is a fetus; yes, but a fetus without one of his most important organs. When the umbilical cord is tied and divided at birth the infant loses touch with his placenta-with that so essential organ of fetal life. With the rupture of the membranes he has already lost touch with the liquor amnii, that fluid environment of antenatal existence which we may call the fetal bydrosphere in contrast with the atmosphere which surrounds the newborn infant. The premature infant, therefore, is a fetus lacking the placenta, membranes, and liquor amnii, which together go to make up the fetal annexa. From the ana. tomical standpoint this is simply a loss; but from the physiological, as we shall see immediately, it is more than a loss, it is a distinct danger to the premature infant. It may be added that immediately after birth he loses another fetal covering, namely, the vernix caseosa, which is carefully and completely removed by the midwifery nurse.

Physiology of the Premature Infant.

While from the anatomical standpoint it may be said that the premature infant is a fetus without the structures which constitute the fetal annexa, the same generalization cannot be applied to the physiology of the child that is untimely born. Physiologically, the premature infant is partly a fetus and partly a newborn child. In him is seen fetal physiology endeavouring to cope with neonatal surroundings, but in him also is seen neonatal physiology hampered in its action by persistent fetal conditions. Some illustrations may be given in proof of this statement.

There is, for instance, the temperature of the premature infant. The fetus in utero has a temperature which is slightly higher than that of the mother; it is from one-tenth to twotenths of a degree (Centigrade) higher than that of the containing uterus; the temperature of the liquor amnii is intermediate. The slight excess of fetal over maternal temperature is to be ascribed to the metabolism which is going on in the fetus independent of that which takes place in the placenta. No doabt fetal oxidation is feeble, possibly it is similar to that going on in hibernating animals; but it is certainly present. Immediately after birth the temperature of the infant falls, a result due in part to the action of the cold atmospheric air, and in part to the evaporation of the liquor amnii from the skin; but in the case of the full-time infant the loss is soon in part compensated by the more active metabolic processes going on in the body of the child, which in their turn are stimulated by the establishment of pulmonary respiration. In a day or two the temperature which is to be the normal for the rest of life may be attained. In the premature infant, on the other hand, the immediate postnatal loss of heat is great, and the temperature falls to a very low level, and, further, it may remain there for a comparatively long time. These facts are no doubt due to several causes, but among them must certainly be reckoned the defective action of the imperfectly expanded lungs, the absence of the thick layer of subcutaneous tissue which is to be found in the fulltime infant, and the feeble metabolism which is going on in the organs and tissues of the premature child. Perhaps, also, the removal of the vernix caseosa and the want of an active heatregulating mechanism may be subsidiary causes of this neonatal hypothermy. The fetus in utero, then, does not require to manufacture heat in order to maintain his body temperature; the newborn infant needs to do so, and generally succeeds well; but the prematurely-born infant is urgently called upon to do so, and has only the tissue metabolism of fetal life slightly reinforced by that of an imperfectly-established pulmonary respiration to aid him in his difficult task. Is it a matter for wonder that he often fails? Neonatai physiology is sadly hampered by persistent fetal conditions. The premature infant, however, can remain in life witin a temperture lower than an adult can, and he can do so for a longer time. Here is the other side, the fetal side, of his physiology coming into play, and endeavouring to cope with neonatal surroundings.

Many other instances of the special characters of the physiology of the premature infant might be given. There is the liver which in the fetus is busily engaged in torming blood and does little in the elaboration of bile; it is suddenly called upon to form much bile, and to continue yet for some time its haematopoietic activities. There is the blood itself which contains more nucleated xanthocytes (erythroblasts) than in the full-time fetus, and very many more red corpuscles. It is not known exactly what special part these elements play in the physiology of fetal life, but the jaundice of the newborn infant, which has been ascribed to breaking down of the xanthocytes, is very common in the prematurely born infant. There is the circulation of the blood : at once its most active area (the placental) is cut off from all connexion with the heart, and that organ is called upon to send a specially large supply to the lungs. This it may or may not succeed in doing, but through the tendency of the foramen ovale and ductus arteriosus to remain patent longer than is normal in the full-time infant, the complete separation of the two currents of blood in the heart and throughout the body is not accomplished; the circulation, therefore, is partly of the fetal and partly of the neonatal type, and it is, in consequence unsatisfactory. With regard to digestion, it is known that pepsine is present in the stomach of the fetus, for fibrine has been digested by a solution made from a scraping of the gastric mucous membrane of an infant that died in birth (craniotomy); the saliva, also, is active to some extent, and there is evidence that the pancreas secretes pancreatine and perhaps trypsine, although not the diastatic lerment; but all these digestive agents are present in small quantity, and although they are doubtless capable of answering the slight calls made upon them by the presence of liquor amnii in the alimentary tract in fetal life they fail of ten in the presence of the more exacting demands resulting from the ingestion of milk by the premature infant. The dry mouth and the weak digestion, and the frequency of gastro-intestinal disorders in the prematurely born are matters of every-doy observation and need not be enlarged on.

Those are some of the signs of the difficulties that arise when the newborn infant attempts, so to eay, to play the part of the full-time child with the functional possibilities of the seven-months fetus. These difficulties are to a large extent removed if the fetus is permitted to remain for a month or two longer in the uterus. In a general sense, they be said to be removed because the fetus matures during the closing period of intrauterine existence, the unborn infant becomes gradually less dependent upon its placenta simply because its tissues gain a greater functional activity. This is true, and yet it is not all the truth. The physiological experi- 
ences of the last three months of fetallife are not simply a continuation of those of the earlier months, differing perhaps in degree but not in kind. Modern research upon the problems of fetal physiology goes to show that in the last trimester there is a functional activity peculiar to the period, an activity which differs distinctly from that which has characterized the middle trimester. It is during this time that fibrous changes are occurring in the chorionic villi, changes which prepare the way for the replacement of the placental activity by that of the various intracorporeal organs of the fetus. The placenta is becoming senile. Further, increased activity is manifest in the kidneys, as is shown by the presence in their tubules of uric acid infarcts, as they are called. But of more importance, probably, than these are the alterations in the transplacental interchanges. This subject, of course, is one of great complexity and difficulty, but new light has been thrown upon it by the chemical analyses of the fetus at different ages, and of the placenta; these are still few in number, but the results are strikingly suggestive.

Functions of the Placenta.

In the first place, there is some proof that leucocytes pass from the placenta to the fetus, for it has been found by Varaldo that the blood of the umbilical vein contains more white corpuscles than does that of the umbilical arteries. Apparently, the matrifugal stream carries more leucocytes to the fetus than the matripetal brings back; some of them must be retained in the tissues of the unborn infant. Possibly the leucocytes carry with them special chemical substances. In the second place, the analyses that have been made of the mineral constituents of the fetus at different ages by Hugounenq and others, show clearly that there is a great fixation of minerals in the last trimester of pregnancy. There is a very marked storing up of iron in this period with a corresponding diminution in the maternal reserve of that metal. Fetal hypersiderosis is accompanied by maternal hyposiderosis. In the last trimester, also, there would appear to be a great transference of potash salts from mother to fetus; in fact the soda salts predominate until the last three months, when the potash salts begin to pass in greater amount. There is also a flush of lime through the placenta in the last weeks, but this is not accompanied by a corresponding increase in the phosphorus transmitted; apparently, therefore, the phosphoric acid which is to combine with the lime in the bones of the fetus passes first, and, as it were, waits for the lime in the fetal tissues. All these observations support the belief that the placenta has great selective powers and is not the simple transmitting organ that it has often been supposed to be; indeed all recent research has gone to enhance the physiological dignity of the placenta, and to elevate it to a high rank among the tissues of the body. It has the power of selecting as well as of transmitting materials to the fetus; its epithelium may even, it is supposed, have a peptonizing effects upon proteids passing through it; it would seem to have the faculty of retaining in its tissues certain microbes, toxins, and poisons which if allowed to pass would harm the unborn infant, resembling in this property the liver in the adult; and it is possible that it has an internal secretion of some value to the maternal organism. The peculiarities of the transplacental interchanges of the last three months of intrauterine life would seem to be specially associated with the formation of red blood corpuscles, of striped muscle and of bone.

Doubtless there are other physiological processes of a distinctive kind going on in utero during this interesting and important epoch of antenatal life; the defences against the assaults of pathogenic microbes are being prepared, the myelination of the nerve tracts in the brain and cord is going on, and the renal epithelium is being made ready for its excretory function; but much research is needed before anything like a complete knowledge of the physiology of fetal existence, and especially of the last three months of it, is available. Enough, however, is known to prove that the premature infant who by reason of his prematurity loses the phrsiological experiences of the last two months of fetal life suffers a great loss. The consequences of the loss are evident during, at, and immediately after birth. Birth is to the premature infant the more or less traumatic transition from an intrauterine to an extrauterine existence.
All transition times are danger times; but Nature always tries to make transitions easy and safe by carefully preparing for them. Natura non facit saltus (Nature makes no leaps) is the old proverb, and the truth of it is very evident in conrexion with the transition of birth. In the case of the premature infant, however, Nature is not allowed time enough to prepare for the sudden changes from a semi-parasitic life inside the uterus to a more or less independent one outside that organ. The absence of the preparatory two or three months with their distinctive physiological events introduces a large degree of extra danger into the occurrences of birth and of the neonatal period. 'The results of this will be stated in the next paragraph.

The Pathology of the Premature Infant.

In its pathology the premature infant stands midway between the fetus and the newborn child. In some respect its morbid processes develop as in the fetus, and in other respects they follow the lines of neonatal pathology. One or two instances may be cited in illustration. A fetus takes diseases, so to say, by the umbilicus; the germs of disease coming to him from his mother pass through the placenta and arrive in his tissues by the way of the umbilical vein and ductus venosus. A newborn infant, on the other hand, receives infection by the more usual avenues of entrance, namely, the gastrointestinal tract, the lungs, and the skin; in his case the umbilical route is effectually closed in part by the ligature of the cord, but more especially by the obliterative changes which take place in the umbilical vessels. In the case of the premature infant the closure of the umbilical arteries and vein is apt to be incomplete, and septic and other pathogenic organisms find their way into the body on account of the persistent permeability of these vessels. The results of the infection are sometimes evident in the immediate neighbourhood of the umbilicus in the form of periumbilical inflanumation of an erysipelatous type, but at other times the umbilical vessels may show no morbid changes save permeability, and the pathological consequences of the microbic invasion may be found in the liver, the heart, or in some distant organ such as the brain. In this respect, therefore, the pathology of the premature infant resembles that of the fetus.

Again, the premature infant is specially liable to attacks of thrush in the mouth, of diarrhoea, and of dyspepsia; these are generally due to infection through the gastro-intestinal canal, predisposed to by the defective secretions of the digestive organs; and in this respect his pathology resembles that of the newborn infant.

Again, the premature infant is like the fetus in the small degree of resistance he offers to pathogenic organisms when they once gain access to his body (this has been demonetrated very clearly in fetal tuberculosis); he is like the newborn. infant, in that many of his morbid states can be traced to injurious pressure acting upon him during his passage through the birth canals.

The result of this combination of the pathology of the fetus and the newborn infant, which is witnessed in the prematurely born child, is greatly increased morbidity and mortality. I need not dwell upon this point; every obstetrician knows from sad experience that it is rare for a prematurelyborn infant to escape illness, and that it is too common for death to occur before what ought to have been his birthday has been reached. Some have placed the morbidity of premature infants as not far from 100 per cent., and the mortality at 50 per cent.; there is only too much reason to believe that in some circumstances this is no exaggerated statement. The prognosis is better when the infant has a weight and a development not widely different from those of a full-time child, when he comes of healthy parents, and when his temperature is not much below that of a normal newborn infant; but, of course, this is saying little more than that his chances of living are greater when he shows fewer signs of prematurity. Too often the premature infant is the off spring of parents who are themselves seriously ill or gravely weakened by toxic agencies; the premature termination of pregnancy is itself one of the signs of these parental states; hence it comes that what may be called normal premature infants are rarely met with. Of course, if the best treatment can be obtained for such a child his chances of survival are greatly increased, 
and it is gratifying among all this prognostic gloom to throw the beam of light which has come recently from two of the large obstetric clinics of Paris (the Maternité de la Charité and the Clinique Tarnier), where the mortality has been reduced to little over 6 per cent.

Enough has been said to prove that the problem that faces the premature infant on his advent into an extrauterine existence is no easy one, and that the risks of failure are great. Let me now devote some sentences to the consideration of the management of the infant born prematurely, dealing rather with general principles than with details.

Management of the Premature Infant.

The management of the premature infant consists in an attempt to attain a perfect adjustment of vital capabilities which have been shown to be generally inefficient to meet requirements which every one must recognize to be peculiarly exacting. The three leading principles which ought to guide us in the performance of this difficult task will be: First, to prolong the most useful and the best features of fetal life aiter birth; secondly, to supply some of the features which cannot be prolonged; and, thirdly, to awaken and strengthen the dormant or inefficient functions peculiar to post-natal existence.

In the first place, then, we ought to try to prolong the fetal phase of life after birth has occurred. We cannot, so to say, return the premature infant to its proper place of abode, the interior of the uterus; but we can with some success endeavour to create an environment for him which shall in some points at least resemble the intrauterine nidus. This environment is found in the incubator or couveuse, or "brooder." A gradual improvement in the construction of the incubator has taken place during the past few years, and the approved model of the present day, such as is to be seen in the Parisian hospitals and such as was on view at the Buffalo Exposition, meets in a fairly satisfactory fashion many of the requirements of the case. A temperature which can be with care kept constantly a little above that which the infant's body should register can thus be obtained, and the little patient can be kept in the dark and shielded from injury. Theoretically the perfect incubator should contain saline solution and not heated air, in order to imitate more closely the nterine interior in pregnancy; and the infant should be bathed in this fluid medium or hydrosphere and suspended in it save only the mouth and nose, but it is doubtful whether this could ever be carried out in actual practice, and one has to be satisfied with an atmosphere (saturated perhaps with moisture) instead of a hydrosphere. The couveuse is a satisfactory means of maintaining the temperature of the child, and it thus prevents one of the great dangers to be apprehended-namely, hypothermy

Some of the features of fetal life cannot, as has been already said, be imitated. Among these, the peculiarities which are due to the presence and activity of the placenta will at once come into the mind. Some of the placental functions are performed by the awakened organs of the premature infant; thus the lungs endeavour to carry on the work of respiration, but the results are often unsatisfactory, and the organs may require to be aided artificially in various ways, as will be referred to immediately. Other of the placental activities, however, cannot be vicariously carried out. For instance, the function of preventing the ingress of germs, pathogenic or non-pathogenic, to the fetal body cannot be done efficiently. The best that can be accomplished is to render and to keep the couveuse as nearly aseptic as may be. Again, it is impossible to imitate the nutritive interchanges which go on between the fetus and the mother by means of the placenta but experience has shown that if the mother is able to nurse, and if the infant is capable of drawing milk from her breast, a great deal can be done to improve the premature child's chances of survival. In the case of the fetus, nourishment passes to the internal organs directly through the blood of the umbilical vein, and reaches the liver at once; in the case of the premature infant that is unable to suck, it has been found to be of advantage to pass predigested food directly into the stomach by means of a tube or to drop it into the mouth with a dropper. This procedure, which has been termed "gavage," imitates, al though not perfectly, the intra- uterine method of feeding, and will often help to save life.

With regard to the composition of human milk as compared with that of the nutritive fluid which passes through the placenta to the fetus in the last weeks of pregnancy, it is necessary to pay some attention. Analyses of the ash of human milk show that it contains a relatively large quantity of potassium salts and of calcium. In respect of these two constituents, therefore, the mother's milk or that of a wetnurse may be said admirably to make up for the loss which the infant has suffered through being deprived of the last two months of intrauterine feeding. Human milk, however, is known to be markedly deficient in iron ; in this respect it differs from the milk of animals, and offers an exception to Bunge's law-resemblance between the ashes of the fetus and those of the milk of the mother animal. This exception has been physiologically explained by the flush of iron which takes place through the placenta in the last three months of fetal life in the case of the human fetus; it has been thought that enough iron is thus stored up in the fetal tissues to counterbalance the deficiency in the mother's milk. This excess of iron is, of course, wanting in the case of the premature infant that has lost the placental interchanges of the last two months of pregnancy.

It, therefore, seemed to me to be necessary to add iron to the food of the prematurely-born child. Theoretically, it might be well if we could give the iron through the skin; but the pharmacologist whom 1 consulted on this point did not regard the plan as feasible. It then seemed to me that we might give it through the mouth in an easily-absorbed and unirritating form. I accordingly tried the effect of the liquor ferri peptonatus, first in the case of full-time infants at the Maternity Hospital, and lately upon a premature infant in private practice. I gave it in doses of $\mathrm{m} v$ thrice daily in a few drops of water between nursings. In no case did the medicine apparently cause any gastric or other disturbance. Of course the experience is too limited to enable us to judge whether the iron will prove a help to enable the premature infant more successfully to cope with the requirements of its new environment ; but, theoretically at least, the giving of it would seem to be justified, and, practically, it does not at any rate appear to do any harm.

If human milk is not available for the prematúre infant's feeding, then, as every medical man knows, the prognosis for the child becomes much graver. The food which is usually given is corv's milk, although asses' milk if obtainable would probably give better results. The amount given ought not to exceed four teaspoonfuls at a time, and the intervals between feedings ought not to be longer than one hour and a-nalf. Sterilization of the milk is a sine quâ non, for the tendency of microbic infection by the gastro-intestinal tract is very great. As to the exact modification of the milk opinions differ, but it would seem to be reasonable to limit the amount of sugar for the powers of the premature infant to digest this substance are small. In these cases, also, the iron would seem to be indicated.

To keep the infant much in the dark is also good, for the light may prematurely stimulate to inco-ordinated action the muscles of the eyeball, and so lead to the production of strabismus.

In the third place our treatment of the premature infant ought to aim at awakening or strengthening the dormant or inefficient functions of its organs. This applies specially to pulmonary respiration, and includes as a rule the resuscitation of the infant at birth, for such infants are generally born in an asphyxiated state. The Schultze swinging movements are not particularly suitable for the premature infant (they increase evaporation from the skin and chilling and may be traumatic), and I recommend rather one of the other plans of exciting respiration, such as the alternate flexing and extending of the trunk of the child as it lies upon the palms of the obstetrician's bands. The infant ought to be wrapped up in cotton wool while this procedure is being carried out. The inhalation of oxygen has been recommended upon the principle that as only a part of each lung is acting it will be advisable to supply to it a larger quantity of oxygen than is contained in ordinary air. The plan has not been specially successful, possibly because the tissues of the premature infant do not lack oxygen, but rather the power of combining with it. 
Urgency of the Problem.

With all care, and with every precaution, the premature infant will often fail to survive birth. This is very specially the case when the prematurity is due to some active or chronic malady of the mother, or to a disease or deformity of the infant himself. The ideal management of the premature infant is, of course, prevention ; and, no doubt, much is possible in this direction. But even if we could banish syphilis and alcoholism and lead poisoning, and the many other causes of the premature ending of pregnancy, there would still remain the infants born as a result of the therapeutic induction of premature labour. If this obstetric operation, which has always been a favourite in British practice, is to hold its own against symphysiotomy and the Caesarean section, it is essential that the infantile mortality and morbidity be greatly reduced. The problem of the premature infant is on this account a very real and a very pressing one. Further, the steady fall in the birth-rate in the British Isles as well as in some foreign countries and in our own colonies, has, so to say, caused an appreciation in the value, economic as well as sentimental, of the premature infant. When it is borne in mind that in England and Wales in 1871 the birth-rate was nearly 35 per I,000 (34.7 was the exact figure), that it had fallen to 29.3 in 1899 , to 28.9 in 1900 , and that it was as low as 28.5 in 1901 , it is evident that there is a pressing need to conserve the lives of the infants that are actually born, even although they are prematurely born. It may not be possible exactly to define their value to the State and the community, but manifestly it is greater now than it was when the birthrate was 35 per 1,000 . The problem of the premature infant is urgent.

AN ANALYSIS OF FORTY-SIX CASES OF CANCER OF THE BREAST WHICH HAVE BEEN OPERATED UPON AND SURVIVED THE OPERATION FROM 5 TO 32 YEARS,

WITH REMARKS UPON THE TREATMENT OF RECURRENT GROWTHS, INCLUDING THE DISEASE OF THE SECOND BREAST, OPERATIVE AND OTHERWISE, ,

By THOMAS BRYANT, M.CH., F.R.C.S., Consulting Surgeon to Guy's Hospital.

Ter paper I ask your attention to this evening should be regarded as a sequel to a communication made by Mr. Marmaduke Sheild on January 25 th, 1898 , to this Society, when I had the honour to occupy the presidential chair; as it was from the interesting collection of facts which he had gathered from varied sources and analysed that I was led to search my own notebooks and to ex tract from them such material as might throw some light upon (I) the prospects of life after primary operations for cancer of the breast; (2) the question of recurrence of the disease at the seat of the primary operation and second breast; and (3) the nature and effects of operation upon the progress of the disease.

It must, however, be steadily borne in mind by the readers of this paper, that the cases tabulated include only such examples of cancer of the breast as have been under my care and have been operated upon, and have survived the primary operation five years and upwards ; for I have always felt that the three years' freedom from recurrent disease alter a primary operation, which has been so dogmatically laid down as a significant indication of a cure of cancerous disease, was not only unreliable but misleading.

In my book on Diseases of the Breast, published in 1887, I satisfactorily showed (page 152) the inaccuracy of such a view, and pointed out that if, after the primary operation for cancer of the breast, 40 patients out of 60 there tabulated died within this three years' limit, there were at least 20 patients who had survived the primary operation from five to ten years, for 4 of these 20 .instances lived for eight or nine years, and 6 for ten years.

In the tables I now bring before you, many instances of much longer survival after the primary operation will be found recorded, and likewise many instances of recurrence of * Read before the Royal Medical and:Chirurgical Society. disease after prolonged periods of immunity which are very striking.

This includes 17 cases of cancer of the breast relieved by operation which are now alive, or have died without evidence of recurrent disease, five or more years after operation; 4 have died, and 13 are living and in good health. Of the 4 which died-

Case ${ }_{15}$ died from an accident, aged 62, five years after the primary peration.

Case 16 from old age, aged 8o, twenty years after operation.

Case 14 from acute jaundice, aged 63, fourteen years afterioperation : and

Case $r_{3}$ from intestinal obstruction due to gall stones, aged 79 , thirteen years after operation. In both of these cases a necropsy was performed and no evidence of recurrent disease was found.

Of the 13 cases which are now alive, I has remained free from recurrence for five years, I for six years, 3 for eight years, 3 for nine years, 2 for ten years, 2 for fourteen years, and i for sixteen years. Taking the whole group of 17 cases together, there was an absence of any evidence of recurrent disease from five to ten years after the primary operation in 9 cases, and from ten to 20 years in 8 cases, 13 of these patients being now alive and apparently well.

I should like here to say that the operation I now do, and have done for many years, is neither the one I was originally taught and have seen practised by my senior colleagueswhich was certainly inadequate-where lymphatic glands were rarely removed, or anything more than the diseased breast itself, with the skin covering it when involved; nor is it the more modern operation known as "Halsted's," and made public in 1894, but which should be known as "Moore's" or Banks's, who advocated the principle of free removal in 1882, not only of the diseased breast with the fat and skin over it in every case, but also of the pectoral muscle, fascia, and lymphoid tissue from the axillary vessels, and which is now known as the complete or adequate operation. My operation is something between the two, but nearer the latter than the former, and I hold with the results before me that it is a complete and adequate measure under the most favourable conditions.

\section{The Operation Adopted.}

My routine operation is to remove the whole gland that is diseased with the skin and fat over the diseased area; when the axillary glands are enlarged to dissect out the axilla and subpectoral spaces, and in every case, for examination purposes, to cut into the axilla, and to take away glands or lymphoid tissue which appears to be suspicious, but otherwise not to dissect it out, $\mathrm{my}$ incision into the axilla skirting the axillary border of the pectoral muscle. I invariably drain the wound through the axilla for the first two or three days.

The pectoral muscle I dissect clean, but do not remove it, although should disease be found to have invaded the muscle, the diseased muscle must be freely taken away. I regard the removal of the muscle as a routine $x$ ceasure to be unnecessary, and the facts I now bring before you tend to support this view, for I am more impressed by accumulating experience that successful results in operations for cancer are more certainly to be secured by an early operation than by "performing tremendous operations upon practically hopeless cases." 1

I may say at once that it was from the careful study of Moore's memorable paper on Inadequate Operations on Cancer, published in 1867 in the fiftieth volume of the Transactions of this Society, that I was led to deviate from the practice I had been taught, and to follow, as far as I thought right, in the lines of Moore's suggestions, which have been, without question, the basis of all recent operative procedures.

In more recent times the principle of free removal of cancerous disease has been well brought before the profession by Sir W. Mitchell Banks in papers of great importance published in 1877,1882 , and 1900 in the British Medical Journal, and it is to him more than to any other writer that Moore's views have become established.

What I regard as a point of more importance than so-called complete or adequate operations is early interference, and in my sanguine hours I have imagined, with Sir Mitchell Banks, what the results would be if all cancers were thoroughly 